\title{
Tangible and Wearable User Interfaces for Supporting Collaboration among Emergency Workers
}

\author{
Daniel Cernea ${ }^{1,3}$, Simone Mora ${ }^{2}$, Alfredo Perez ${ }^{2}$, Achim Ebert ${ }^{1}$, Andreas Kerren ${ }^{3}$, \\ Monica Divitini ${ }^{2}$, Didac Gil de La Iglesia ${ }^{3}$, Nuno Otero ${ }^{3,4}$ \\ ${ }^{1}$ University of Kaiserslautern, Germany \\ \{cernea, ebert\} @cs.uni-kl.de \\ ${ }^{2}$ Norwegian University of Science and Technology, Norway \\ \{simone.mora, perezfer, monica.divitini\}@idi.ntnu.no \\ ${ }^{3}$ Linnaeus University, Sweden \\ \{andreas.kerren, didac.gil-de-la-iglesia, nuno.otero\}@lnu.se \\ ${ }^{4}$ University of Minho, Portugal
}

\begin{abstract}
Ensuring a constant flow of information is essential for offering quick help in different types of disasters. In the following, we report on a workin-progress distributed, collaborative and tangible system for supporting crisis management. On one hand, field operators need devices that collect information-personal notes and sensor data-without interrupting their work. On the other hand, a disaster management system must operate in different scenarios and be available to people with different preferences, backgrounds and roles. Our work addresses these issues by introducing a multi-level collaborative system that manages real-time data flow and analysis for various rescue operators.
\end{abstract}

Keywords: Wearable tangible device, collaborative crisis management.

\section{Introduction}

Humans, despite technological and scientific advances, are still vulnerable in the face of natural disasters. It is therefore essential to provide effective management and quick aid in such scenarios $[8,15]$. Providing up-to-date data, ensuring a constant flow of information, organizing and coordinating rescue units and reaching the people in need are the core factors for ensuring disaster management and offering quick help. This paper presents an exploratory design study on tangible user interfaces for improving coordination in crisis management.

Designing novel Disaster Management Information Systems (DMIS) poses unique challenges [1,2]. Multiple publications have focused on interaction techniques for crisis management systems, capturing vital aspects in the areas of multitouch [3, 17] or gesture interaction $[1,4]$, with a special emphasis on map-based approaches. At the same time, solutions have been devised that aid the cooperation and interaction of disaster managers and unit operators in the settings of a mobile command post connected to mobile devices [18]. Still, while mobile devices like tablets and smart phones would seem ideal, the need for additional information about the environment

In Proceedings of the 18th CRIWG Conference on Collaboration and Technology (CRIWG '12), pages 192-199, volume 7493 of LNCS, Duisburg, Lower Rhine region, Germany, 2012. Springer.

(c) Springer, 2012. This is the authors' version of the work. It is posted here by permission of Springer for your personal use. Not for redistribution. The original publication is available at www.springerlink.com. Springer Verlag. 
$[6,7]$ and specific operation conditions has lead to scenario-fitted approaches, where field operators employ handheld [5] and wearable devices [14].

We aim at exploring how tangible interaction impact on crisis management and we propose a prototypical system implemented by a tabletop interface for team coordinators and disaster managers sitting in a control room, and by a wearable interface attached to each field agent's forearm. In the following sections, we describe the features and functionality of our tangible collaborative system. Next, we focus on the evaluation of our system by a group of experienced rescue workers and discuss our findings. Finally, we conclude highlighting the major findings, their implications and plans for future work.

\section{User studies and scenario}

In this section we highlight a scenario for the proposed crisis management system. The scenario has been developed building on observations and interviews with emergency workers performed during a three-day simulation of a massive disaster held in Italy in 2011. Scenarios included flooding, earthquake and a massive car jam. Rescue workers were deployed to find and rescue persons (i.e. actors impersonating injured persons) in a physical environment that resembled a real disaster; team coordinators and disaster managers were directing operations from a control room. Teams included rescue units, civil protection, police, responder for hazardous and chemical contamination, dog rescue units. One of the paper's authors shadowed workers assigned to different roles during the three days in order to gain an understanding of procedures and technology in use for coordination during a crisis response.

Results from the study show that agents still largely rely on handheld transceivers (i.e. walkie-talkies) to communicate among each other and with the team coordinators. Once the rescue and management operations are underway, the field agents are given instruction by coordinator through radio broadcasts. At the same time, field workers have to communicate back information like their position, environmental data (temperature, humidity, air quality) in a half-duplex communication. As this can be only done in a qualitative way, often their information can get biased or distorted [13]. Additionally, the units on the field need to remember and execute the tasks and commands assigned to them by the coordinators. Meanwhile, coordinators in meeting rooms need to transcribe the radio communication, as well as annotate and update on a map the positions of the teams and data they have collected. Building on results from the study we have developed a scenario to show how the use of tangible and wearable technology might impact on the work practice.

\section{The Scenario}

Scene: EM coordinators in a mobile command center - Disaster managers activate an emergency response gathering around a tabletop in a mobile unit (Figure 1, left). They explore a map of the disaster and decide where to deploy the emergency 
units. There are different units depending on the specific disaster to consider (e.g. flood, fire, earthquake, etc.).

Scene: EM workers on the disaster scene - Workers arrive to the crisis scene. Wearable devices are consulted in order to identify context information (the place they have been assigned, noise level and temperature) and tasks to carry out (Figure 1, right). A worker has received a notification requesting his reallocation in a different sector. Once having performed the task he acknowledges the conclusion by interacting with his wearable device.

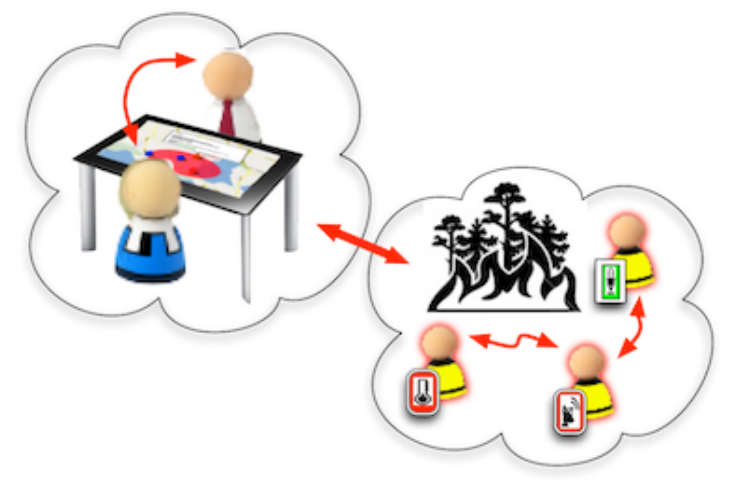

Fig. 1. The dimensions of collaboration supported by the emergency management system.

\section{Design and User Interaction}

The proposed solution we have designed consists of two main elements with wireless capabilities for information sharing. These elements are a tabletop unit used to manage and coordinate the different field units, and a wearable unit in the form of a wristlet to be used by workers on the field.

\subsection{Tabletop Unit}

The proposed Mobile Command Center (MCC) has the role of supporting the decision making process of the unit coordinators. The tabletop allows multiple users to interact with the map of the affected region, as well as gather and analyze data a constant data stream (real-time information from wearable devices). Furthermore, the tabletop can receive and display new information about the crisis situation as it pours in from the public to the rescue services (e.g. dispatcher). At the same time, coordinators have the possibility to independently send messages to the field units to inform them about new developments or give instructions on how to proceed.

The initial step for managing a crisis is the registration of the event in the MCC. To do so, colored marker objects (Figure 2) are used, which are meant as an efficient and intuitive way to manipulate the location and type of the reported disaster. The color of the marker encodes the type of the disaster, allowing other rescue services to be automatically informed. 
Furthermore, the coordinators can interact with the marker objects to update the information about the disaster. Once the changes to the event are made, the marker object can be removed from the tabletop to avoid occlusion. Placing the marker on the same event site allows users to customize or delete the corresponding disaster information. After an event is registered, the MCC uses its wireless Internet connection to query the database of the rescue services, in order to get additional information about the rescue efforts. Aiming to support readability and collaboration, the users have the possibility to rotate any text or marker.

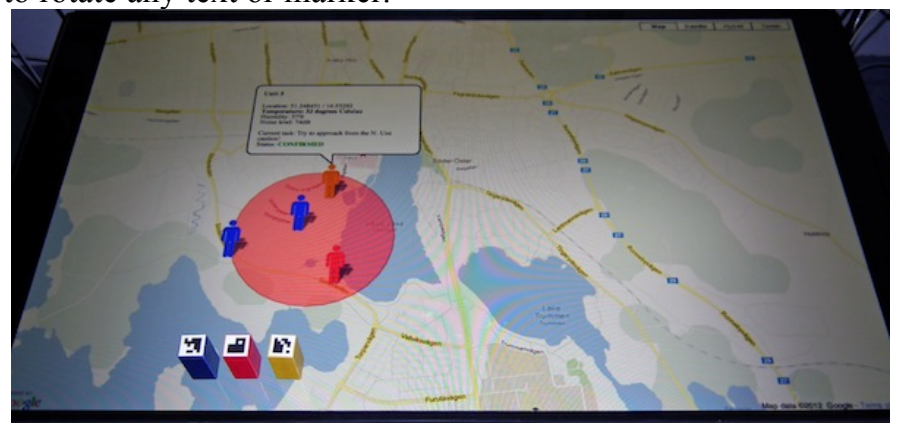

Fig. 2. Tabletop running the MCC system. Colored marker objects enable the user interaction with information from the field.

One of the most important tasks of the MCC is the communication and coordination of the units on the field. In this sense, each wearable tailored device sends a constant stream of data to the tabletop via a wireless Internet connection. This information is evaluated at the MCC and displayed in real-time for each unit. The collected environment data can be collaboratively and interactively visualized. Coordinators can also access a priority list for sent messages that highlights all the tasks and their current status (received, confirmed or executed).

\subsection{Wearable Unit}

The wearable device is to be worn on the field worker's arm (Figure 3). User interaction is supported by a LCD color display and a proximity-activated button located on the armband that holds the device. Interaction with the device is designed to disrupt rescue operation as little as possible: high-contrast colors have been chosen in order to enhance screen readability under direct sunlight, while the proximity button can be activated even wearing gloves.

Once activated the device start displaying the following information: GPS coordinates and ground speed, environmental temperature, noise level, the task that the user is assigned to (pre-defined on the tabletop unit), a green/red bar indicating whether the assigned task has been completed or not

GPS and environmental data are also transmitted to the tabletop unit via a wireless connection. We designed the device to be based on modules so different type of sensor and network adapters can be adopted to address the precise disaster need. A proximity-activated button is located on the device armband. By brushing the armband the 
user can acknowledge the coordination unit that a task has been completed. The status bar on the display turns green to confirm to the user that the task-completion message has been sent to the tabletop unit and the device is ready to receive a new task.

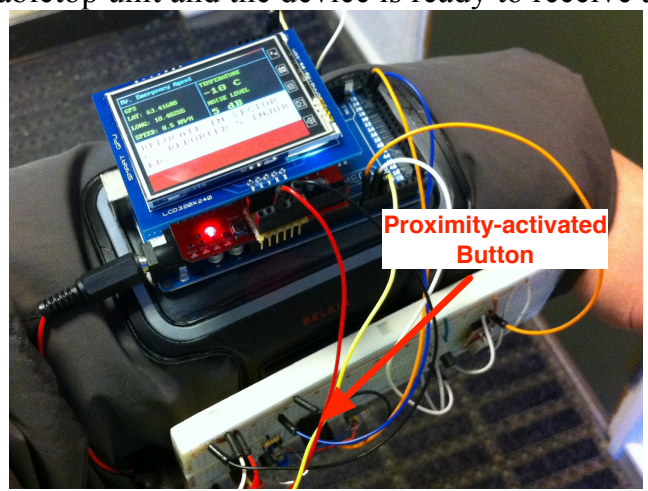

Fig. 3. Proximity-activated button is positioned on the armband. The device notifies the user about a new task received with audio and haptic feedbacks.

\section{$4 \quad$ Architecture and Implementation}

\subsection{Tabletop Unit}

The MCC system that corresponds to the different coordinators of the disaster management efforts, was implemented on a MultiTouch Cell tabletop ${ }^{1}$. The core ideas behind this system include the support for collaboration between multiple unit coordinators, as well as the mobility of a vehicle-mounted tabletop as a communication and management platform. The software running on the tabletop is written in Adobe Flash ActionScript 3. Additionally, the Google Maps API for Flash ${ }^{2}$ has been used to allow the manipulation of interactive maps.

\subsection{Wearable device}

The first prototype of the wearable unit has been developed using an Arduino Mega board ${ }^{3}$ as central module. Both hardware and software have been developed for this work. User interaction is assisted by a 2.5" LCD display, sound and haptic notifications are provided by a buzzer and a small vibration motor. The user can send input to the device using a proximity-activated button working in infrared light, which allows for use also wearing gloves. Location tracking is enabled by a 66 channels GPS chip, which senses the user's location 5 times per seconds. Network communication is available via an XBee S1 adapter, according with specifications ${ }^{4}$ it provides a $120 \mathrm{mt}$

\footnotetext{
${ }^{1}$ Multitouch Cell. http://multitouch.fi/products/cell/

${ }^{2}$ Google Maps API for Flash. https://developers.google.com/maps/documentation/flash/

${ }^{3}$ Arduino Mega. http://arduino.cc/en/Main/ArduinoBoardMega

${ }^{4}$ XBee. http://www.digi.com/xbee/
} 
communication range with low power demand, the Xbee socket on the board is also compatible with other adapters, as for example the Xbee-PRO 868 capable of communication range up to $80 \mathrm{~km}$ (outdoor, line-of-sight). Tests have shown that the system can be powered up to 90 minutes depending on environmental temperature and the strength of the GPS signal. Increased autonomy can be provided with arrays of 9V batteries, trading autonomy against device weight and size. The software running on the MEGA board has been developed using the Arduino 1.0 SDK, the TinyGPS library ${ }^{5}$ has been used to parse data from the GPS chip.

\section{Formative Evaluation}

To gather feedbacks, we recorded a video to illustrate the scenario and usage of the prototypes. After having watched the video, seven testers were asked to fill in a questionnaire using a Likert-type scale and open questions. Questions were elaborated around five areas considered important for assessing the technology acceptance of the system: scenario and problem analysis, system architecture, user interaction (overall), wearable device interaction, tabletop device interaction. We showed the video during two videoconference sessions and collected results from the questionnaire as well as informal discussions. Participants of our evaluation were both IT technical staff working for emergency response forces and the EM workers themselves. Results and implication for design are reported below.

Scenario and Problem Analysis - Results from the evaluation show a good user acceptance of the system, the workers clearly stated their interest in using the system frequently. The scenario we are addressing is also acknowledged as well grounded since it often occurs during emergencies.

System Architecture - The different devices and functionalities of the system were properly interpreted by the interviewees, and the overall functionalities were considered coherent. The data types the system is sensing and displaying (location, noise, temperature) to enhance collaboration are considered meaningful during crisis management. Moreover, the users have shown a strong interest in allowing for tailoring the system by adding more sensing capabilities to meet certain crisis scenarios.

User Interaction - Although the efforts made in creating a user-friendly design, the system is still considered somehow complex and we need to do more work on both the wearable and the tabletop prototype. On one side the system is considered easy to use, but a need for a tutor or course to get started with it is suggested. None of the interviewees considered it feasible for the user to set-up the system and to configure networking functionalities.

Wearable Device Interaction - The wearable unit is currently considered too big and heavy to be successfully employed in real rescue operations; an improved hardware design and the production of custom electronic parts could drastically reduce the device dimensions and weight. Also further user studies and design workshops should be carried out in order to choose where to position the wearable unit on the user's

\footnotetext{
${ }^{5}$ TinyGPS, http://arduiniana.org/libraries/tinygps/
} 
body ("Forearms and hands are needed to be free for movements and to raise weights"). Feedbacks reveal that on the field, users might want to have more control on data collected by sensors, for example by being able to set the granularity or the sample frequency. Some comments suggested to allow the device to display sensor data from nearby units worn by colleagues: "It would be useful to have the data displayed for all units, all the time"; this is intended to give to the field agents extended awareness of the local environment and potential dangers.

Tabletop Device Interaction - The tabletop unit is considered helpful, but interviewees stated the need to improve both data visualization and interaction modalities. Discussions with the interviewees have shown that users would benefit from an extended range of physical artifacts and gestures to interact with the user interface. Also we should investigate additional visualization metaphors (heat maps, tag clouds) in presence of a huge amount of data and offer further solutions for avoiding occlusions.

\section{Conclusions}

In this paper we presented a scenario-based development of a distributed tangible system to support disaster management. The potential of the system is highlighted in a formative evaluation that involved emergency workers and IT consultants with expertise in IT systems for crisis management. The preliminary evaluation acknowledged the system as useful, although future works are needed to improve the design particularly in the usability area. In the future, we plan to build on the evaluation results and to involve emergency workers in participatory design sessions in order to elicit additional information and inform the development of new prototypes.

\section{$7 \quad$ References}

1. F. Wang, R. Wen and S. Zhong. Key Issues in Mapping Technologies for Disaster Management, Proc. 2nd International Conference on Information Engineering and Computer Science (ICIECS), pp.1-4, 2010.

2. M. Frassl, M. Lichtenstern, M. Khider and M. Angermann. Developing a System for Information Management in Disaster Relief - Methodology and Requirements, Proc. 7th International ISCRAM Conference, 2010.

3. K. Nebe, F. Klompmaker, H. Jung and H. Fischer. Exploiting new interaction techniques for disaster control management using multitouch-, tangible- and penbased-interaction, Proc. 14th International Conference on Human-Computer Interaction: interaction techniques and environments - Volume Part II (HCII'11), Julie A. Jacko (Ed.) Springer-Verlag, Heidelberg, pp. 100-109, 2011.

4. E. Artinger, T. Coskun, M. Schanzenbach, F. Echtler, S. Nester and G. Klinker. Exploring Multi-touch Gestures for Map Interaction in Mass Casualty Incidents, 3. Workshop zur IT-Unterstützung von Rettungskräften im Rahmen der GIJahrestagung Informatik, 2011.

5. M. Mecella, M. Angelaccio, A. Krek, T. Catarci, B. Buttarazzi and S. Dustdar. WORKPAD: an Adaptive Peer-to-Peer Software Infrastructure for Supporting 
Collaborative Work of Human Operators in Emergency/Disaster Scenarios. Proc. International Symposium on Collaborative Technologies and Systems (CTS '06). IEEE Computer Society, 2006, pp.173-180.

6. C. Fischer and H. Gellersen. Location and Navigation Support for Emergency Responders: A Survey. IEEE Pervasive Computing 9, 1 (January 2010), pp. 38-47.

7. K. Lorincz, D.J. Malan, T.R.F. Fulford-Jones, A. Nawoj, A. Clavel, V. Shnayder, G. Mainland, M. Welsh and S. Moulton. Sensor Networks for Emergency Response: Challenges and Opportunities. IEEE Pervasive Computing 3, 4 (October 2004), pp. 16-23.

8. J. Bergs, D. Naudts, N. Van den Wijngaert, C. Blondia, I. Moerman, P. Demeester, J. Paquay, F. De Reymaeker and J. Baekelmans. The ADAMO project: Architecture to support communication for emergency services, Pervasive Computing and Communications Workshops (PERCOM Workshops), 8th IEEE International Conference on, pp.382-387, 2010.

9. K. Tierney and J. Sutton. Cost and culture: Barriers to the adoption of technology in emergency management. RESCUE Research Highlights, 2005.

10. B.S. Manoj and A.H. Baker. Communication challenges in emergency response. Commun. ACM 50, 2007, pp. 51-53.

11. S.D. Scott, K.D. Grant and R.L. Mandryk. System guidelines for co-located, collaborative work on a tabletop display. Proc. of ECSCW'03, Kluwer Academic Publishers, 2003, pp. 159-178.

12. A. Tang, M. Tory, B. Po, P. Neumann and S. Carpendale. Collaborative coupling over tabletop displays. Proc. SIGCHI conference on Human Factors in computing systems (CHI '06), ACM, 2006, pp. 1181-1190.

13. A. Monares, S.F. Ochoa, J.A. Pino, V. Herskovic, J. Rodriguez-Covili, and A. Neyem. 2011. Mobile computing in urban emergency situations: Improving the support to firefighters in the field. Expert Syst. Appl. 38, 2 (February 2011), pp. 1255-1267.

14. D. Curone, G. Dudnik, G. Loriga, J. Luprano, G. Magenes, R. Paradiso, A. Tognetti, A. Bonfiglio. Smart Garments for Safety Improvement of Emergency/Disaster Operators. Proc. the 29th Annual International Conference of the IEEE EMBS Cité Internationale, Lyon, France, August 23-26, 2007.

15. A. Meissner, W. Zhou, W. Putz, J. Grimmer. MIKoBOS - a mobile information and communication system for emergency response. Proc. 3rd Intl. Conference on Information Systems for Crisis Response and Management, pp.92-101, 2006.

16. J. Lachner, H. Hellwagner: Information and Communication Systems for Mobile Emergency Response. UNISCON 2008: pp. 213-224.

17. T. Bader, A. Meissner, R. Tscherney. Digital map table with Fovea-Tablett: Smart furniture for emergency operation centers. Proc. 5th International Conference on Information Systems for Crisis Response and Management, pp. 679-688, 2008.

18. T.Piazza, H.Heller, M.Fjeld. CERMIT: Co-located and Remote Collaborative System for Emergency Response Management. Proc. SIGRAD 2009, Visualization and Design, Goeteborg, Sweden, pp. 12. 